\title{
Coherent and incoherent multiple scattering
}

\author{
Julien Chabé, ${ }^{1,2}$ Mohamed-Taha Rouabah,,${ }^{1,3}$ Louis Bellando, ${ }^{1}$ Tom Bienaimé, ${ }^{1,4}$ Nicola Piovella, ${ }^{5}$ \\ Romain Bachelard, ${ }^{6}$ and Robin Kaiser ${ }^{1}$ \\ ${ }^{1}$ Université de Nice Sophia Antipolis, CNRS, Institut Non-Linéaire de Nice, UMR 7335, F-06560 Valbonne, France \\ ${ }^{2}$ Laboratoire Lagrange, UMR7293, Université de Nice Sophia-Antipolis, CNRS, Observatoire de la Côte d'Azur, \\ Parc Valrose, Bâtiment H. FIZEAU, 06108 Nice, France \\ ${ }^{3}$ Laboratoire de Physique Mathematique et Physique Subatomique, Université Constantine 1, Route Ain El Bey, 25017 Constantine, Algeria \\ ${ }^{4}$ Laboratoire Kastler Brossel, CNRS, ENS, UPMC, 24 rue Lhomond, F-75005 Paris, France \\ ${ }^{5}$ Dipartimento di Fisica, Università degli Studi di Milano, Via Celoria 16, I-20133 Milano, Italy \\ ${ }^{6}$ Instituto de Física de São Carlos, Universidade de São Paulo, 13560-970 São Carlos, São Paulo, Brazil
}

(Received 12 February 2014; published 21 April 2014)

\begin{abstract}
We compare two different models of transport of light in a disordered system with a spherical Gaussian distribution of scatterers. A coupled dipole model, taking into account all interference effects, is compared to an incoherent model, using a random walk of particles. Besides the well-known coherent backscattering effect and a well pronounced forward lobe, the incoherent model reproduces extremely well all scattering features. In an experiment with cold atoms, we use the momentum recoil imparted on the center of mass of the sample as a partial probe of the light-scattering properties. We find that the force acting on the center of mass of the atoms is not well suited to exhibit the coherence effects in light propagation under multiple-scattering conditions.
\end{abstract}

DOI: 10.1103/PhysRevA.89.043833 PACS number(s): 42.50.Nn, 42.25.Bs, 03.65.Yz, 32.80.Qk

\section{INTRODUCTION}

Coherence effects in the transport of wave in disordered systems are at the heart of many phenomena in various areas of research. In this work, we focus on light propagation, even though many if not most features could be extended to different types of waves, be it acoustic waves, plasmons, heat, antennas, or matter waves such as electrons or ultracold atoms. In mesoscopic physics, coherences are fundamental for weak [1,2] and strong localization of light [3-11]. Coherences are also at work in the universal conductance fluctuations [12], modifications of the local density of states [13], or extraordinary optical transmission [14]. Cooperative emission of light as discussed by Dicke in the 1950s [15] and the response of a cloud of cold atoms excited by an external laser [16] are also based on coherence effects, such as quantum memories using electromagnetic induced transparency in three-level systems [17]. The recent development of ultrastable atomic clocks also relies on optical transitions in the presence of many atoms [18] where the impact of residual multiple scattering deserves particular attention.

Whereas a rigorous investigation of light propagation in the presence of many scatterers requires taking into account the effects of interferences, in most situations interference effects can be neglected and a radiative transfer equation is thus often used in optics, allowing a practical approach to scattering of light in complex media. In this work, we compare an approximate model, based on such an incoherent random walk of photons, to a more rigorous approach, based on a microscopic coupled dipole model. Cold atoms provide an excellent medium to study these fundamental effects. This ensemble of resonant point scatterers are free of defects and absorption and the coupled dipole model is expected to provide an excellent description of the scattering properties of this sample (despite some limitations, which will be discussed at the end of the work). We stress that despite the apparent simple situation, no analytical result is available for a disordered system of $N$ coupled dipoles. This problem has the full complexity of a true many-body problem [19] and one thus needs to resort to numerical or experimental answers to this question. Indeed, if the amplitudes of $N$ coupled dipoles are to be found, this amounts to solving $N$ coupled equations, even though this can be considered as a linear optics problem described by the propagation of a low intensity or single photon field.

This paper is constructed as follows. First we present the results of numerical simulations using both a random walk of photons (RW) and a coupled dipole approach (CD), where the many-body problem is solved by tracing over the photon degrees of freedom. Then we compare the numerical results from both the RW and CD models to experimental data obtained by monitoring the radiation pressure force on the center of mass of the atomic cloud as a probe of the emission diagram.

\section{RANDOM-WALK MODEL}

The radiative transfer equation $[20,21]$ is a very useful and often used model to describe the multiple-scattering regime that takes place in optically thick media. In such a system one photon undergoes a large amount of independent scattering events from randomly positioned particles and therefore interferences between different paths are supposed to be smeared out by the disorder. Then, the transport properties are mostly independent from the wave nature of light and from the particular nature of the scatterers. Such an incoherent model of multiple scattering of photons undergoing a random walk inside the sample provides a good description of these systems, in particular when considering configuration averaged signals. For this incoherent random-walk model, we perform a Monte Carlo simulation where photons are isotropically scattered after a distance $l_{s c}(r)=1 /\left[n(r) \sigma_{s c}\right]$, where $\sigma_{s c}$ is the scattering cross section and $n(r)=n_{0} e^{-r^{2} /\left(2 \sigma_{R}^{2}\right)}$ is the spatial density distribution of the cold atom cloud 


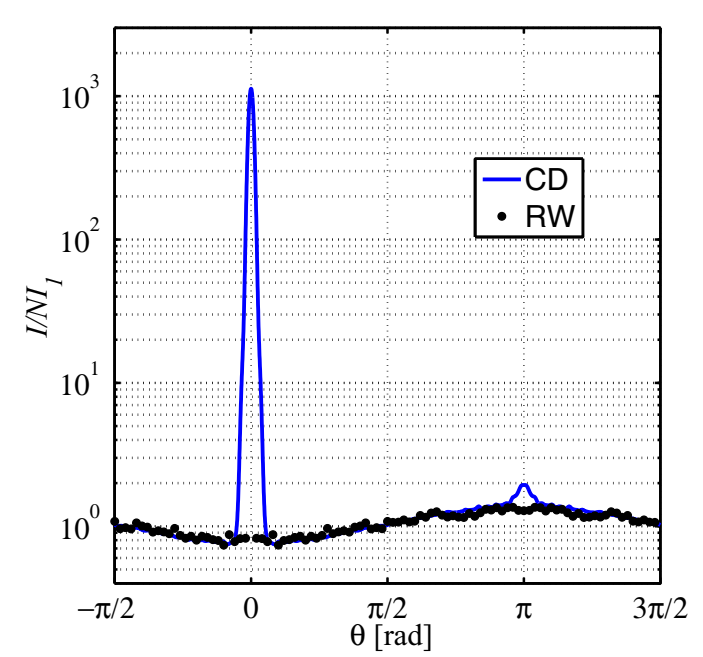

FIG. 1. (Color online) Emission diagram (log scale) normalized to the independent $\mathrm{N}$ atom case, for the RW approach (black points) and the CD model (blue line) with $b=8$.

considered in this work, well described by a spherical Gaussian distribution of size $\sigma_{R}$ and center density $n_{0}$. The optical thickness along one line of sight across the center of the cloud is defined as $b=\int n(0,0, z) \sigma_{s c} d z=\sqrt{2 \pi} n_{0} \sigma_{s c} \sigma_{R}=$ $\sqrt{2 \pi} \sigma_{R} / l_{s c}(r=0)$. By integrating the extinction over the whole transverse size of the cloud $\left(\int d x d y e^{-b(x, y)}\right)$, it is possible to obtain the total extinction of an incident flux of photons, corresponding to a total scattering cross section for this random-walk model given by $\sigma_{R W}=2 \pi \sigma_{R}^{2} \operatorname{Ein}(b)$, where $\operatorname{Ein}(b)$ is the entire function $\operatorname{Ein}(b)=\int_{0}^{b}\left(1-e^{-t}\right) d t / t \underset{b \rightarrow 0}{\rightarrow}$ $b$. To compute the emission diagram, averaged over the azimuthal angle $\phi, I(\theta)$ for this specific geometry, we simulated the trajectories of $10^{5}$ photons arrived along the $z$ axis, over a uniform disk of radius $R=4.36 \sigma_{R}$ for a sample of $b=8$. With $\approx 28.2 \%$ of this "plane" wave diffused, this corresponds to a scattering cross section for the Gaussian cloud $\sigma_{R W} \approx 16.8 \sigma_{R}^{2}$, very close to the analytical value $2 \pi \sigma_{R}^{2} \operatorname{Ein}(8) \approx 16.7 \sigma_{R}^{2}$. For each photon, we record the direction of emission obtaining the angular emission diagram $I_{R W}(\theta)$ (see Fig. 1).

\section{COUPLED DIPOLE MODEL}

We now compare this random-walk approach to a model of coupled dipoles that accounts for the interference between the radiation of all the atoms. Even though light scattering in three dimensions requires to take into account the polarization as well as the near-field dipole-dipole coupling, a better comparison to our isotropic random-walk approach is obtained using a scalar model for the dipole-dipole coupling. In the steady-state regime, the $N$ dipoles with amplitudes $\beta_{j}$ and position $\mathbf{r}_{j}$, illuminated by a quasiresonant plane wave with wave vector $\mathbf{k}_{0}=k_{0} \hat{z}$, obey the equation [16]

$$
\left(i \delta-\frac{\Gamma}{2}\right) \beta_{j}=i \frac{\Omega_{0}}{2} e^{i \mathbf{k}_{0} \cdot \mathbf{r}_{j}}+\frac{\Gamma}{2} \sum_{m \neq j} \beta_{m} \frac{\exp \left(i k_{0}\left|\mathbf{r}_{j}-\mathbf{r}_{m}\right|\right)}{i k_{0}\left|\mathbf{r}_{j}-\mathbf{r}_{m}\right|}
$$

where $\omega_{a}$ is the resonance frequency, $\delta=\omega_{a}-\omega_{k_{0}}$ is the laser detuning, $\Gamma$ is the atomic transition linewidth, $\Omega_{0}=d E_{0} / \hbar$ is the Rabi frequency, and $d$ is the dipole matrix element. This many-body problem with interference has been derived from a quantum formalism where a single photon is shared between all atoms through a superposition of states [16], but also from a classical approach where the atoms are considered as oscillators [22]. The relevant parameters to describe light scattering in dilute clouds of two-level systems is the resonant optical thickness of the cloud, which is given by $b_{0}=2 N /\left(k_{0} \sigma_{R}\right)^{2}$ with an on-resonant scattering cross section for a single atom given in the scalar model by $\sigma_{s c}=\lambda^{2} / \pi$. The detuning dependent optical thickness then reads $b=b(\delta)=$ $b_{0} /\left(1+4 \delta^{2} / \Gamma^{2}\right)$. From (1), the far-field intensity in a direction $\mathbf{k}$ and at a distance $r$ can be calculated using [23]

$$
4 \pi r^{2} I(\hat{\mathbf{k}})=\hbar \omega_{k_{0}} \Gamma \sum_{j, m=1}^{N} \beta_{j} \beta_{m}^{*} e^{-i k_{0} \hat{\mathbf{k}}\left(\mathbf{r}_{j}-\mathbf{r}_{m}\right)} .
$$

All numerical CD results data shown in this paper have been obtained using an average over 20 different configurations of the atomic distribution. We point out that the interferences are not only present in the emission term (2) (Rayleigh scattering), but already in the steady-state value of the atomic dipoles (1). We can now compare the angular emission diagrams obtained from the coherent $\mathrm{CD}$ equations to those from the incoherent RW model. In Fig. 1 we have normalized the emission diagram of the CD model to the emission diagram of $N$ independent atoms (obtained by using a very large sample size with vanishing optical thickness). The emission diagram of the RW model has been normalized such that

$$
\frac{P_{C D}}{P_{R W}}=\frac{\sigma_{C D}}{\sigma_{R W}},
$$

where $P_{C D}$ (respectively $P_{R W}$ ) is the scattered power of the $\mathrm{CD}$ (respectively RW) model for the same incident intensity. $P_{C D}$ and $\sigma_{C D}$ can be obtained from integration of the emission diagram $\int I(\hat{\mathbf{k}}) d \hat{\mathbf{k}}$ or also from $\sigma_{C D}=$ $-\frac{4 \pi}{k_{0}^{2}} \frac{\Gamma}{\Omega_{0}} \operatorname{Im}\left[\sum_{j} \beta_{j} e^{-i \mathbf{k}_{0} \cdot \mathbf{r}_{j}}\right]$ [23]. The total cross section for the incoherent scattering $\sigma_{R W}$ is obtained from the analytical expression given above. For the parameters in Fig. $1(b=8$, $\left.\sigma_{R}=20 / k_{0}, \delta=0\right)$, we obtain $\sigma_{C D}=24.5 \sigma_{R}^{2}$ and $\sigma_{R W}=$ $16.7 \sigma_{R}^{2}$. The difference in these total scattering cross sections is mainly explained by the additional strong forward lobe in the $\mathrm{CD}$ (see Fig. 1), and can also be understood as the origin of the extinction paradox [23].

As one can clearly see in Fig. 1, the RW model in this multiple-scattering regime is very close to the coherent $\mathrm{CD}$ model, except for the coherent backscattering cone and the forward lobe, with an angular width given by the inverse size of the sample. The quantitative agreement between the RW and the CD model in all angles except the forward and backward direction suggests that interferences indeed appear washed out under multiple-scattering conditions, as naively expected. We note however that interferences might nevertheless be relevant in multiple scattering, when spatially dense samples are considered and one expects to approach the Anderson localization transition or when going beyond the average emission diagram in steady state. 


\section{EXPERIMENTAL RESULTS}

The differences between coherent and incoherent scattering have been addressed in the past, including experiments on cold atoms, with the possibility to exploit the detuning as a relevant control parameter. The enhanced backscattering cone, visible only in the CD model and absent in the RW model, has been extensively studied more than ten years ago [24]. The most important difference between coherent and incoherent multiple scattering with a Gaussian-shaped sample is the pronounced forward lobe. Detecting light scattered in a direction close to the incident radiation is notoriously difficult as most detecting schemes will be saturated by the large incident radiation. An elegant technique has been used in [25] but has not been implemented so far with atomic clouds. An alternative approach to indirect probe features of cooperative scattering by laser cooled atoms has been used in the single-scattering limit in [26], where the modification of the atomic motion induced by light scattering has been used as a measure of cooperative scattering. It is therefore interesting to investigate how the radiation pressure force on the center of mass of the atoms extends into the multiple-scattering limit.

We have therefore used the same experimental setup and protocol as in [26], and used values of the laser frequency around the atomic resonance $(\delta \approx 0)$, thus entering the multiple-scattering limit. We apply the following experimental procedure to probe the coherence of the multiple-scattering regime in our cold atom cloud. First, we load a magneto-optical trap (MOT) with $3 \times 10^{7}$ atoms of ${ }^{87} \mathrm{Rb}$ in $50 \mathrm{~ms}$ using the setup described in [26]. We then apply a 50-ms temporal dark MOT period where the intensity of the repumping laser is reduced by a factor of 10 and the cooling laser is tuned to $-10 \Gamma$ from the $F=2 \rightarrow F^{\prime}=3 \mathrm{D}_{2}$ line. This allows us to compress the cloud and to produce a smooth Gaussian-shaped distribution of atoms. To control the optical thickness at the end of this dark MOT period, the repumper detuning is varied between $-7 \Gamma$ and $-2.5 \Gamma$, keeping the desired amount of atoms in the $F=2$ state without affecting size $\left(\sigma_{R}=270 \mu \mathrm{m}\right.$, $\left.k \sigma_{R} \approx 2 \times 10^{3}\right)$, shape, and temperature $(\sim 20 \mu \mathrm{K})$ of the cloud. Here we focus on moderate values of optical thickness, as this allowed for more systematic data without drifts of the relevant parameters. We then switch off all laser beams and magnetic-field gradients, leaving the atoms in free fall. We then apply a horizontal, circularly polarized "pushing" beam, tuned close to the $F=2 \rightarrow F^{\prime}=3$ transition for $50 \mu \mathrm{s}$. The pushing beam has a waist $w_{0}=12 \mathrm{~mm}$ and its carefully calibrated intensity is adjusted to have a saturation parameter $s=8 \times 10^{-2}$. Each atom in the $F=2$ state scatters on average 80 photons. Such a small number of scattered photons prevents any depumping effect into the $F=1$ state during the pushing process. After a time-of-flight expansion of $12 \mathrm{~ms}$, we image the position of the atomic cloud via standard off-resonant (detuning $\delta \approx-2 \Gamma$ ) absorption scheme. The absorption image gives the position of the center of mass of the atomic cloud after time of flight and thus the average radiation pressure force. Each experimental point (see Fig. 2) is an average over ten realizations. We normalize the measured average radiation pressure force by the single atom force $F_{1}$, where $F_{1}$ is computed without any adjustable parameter from the measured intensity of the pushing beam (known within

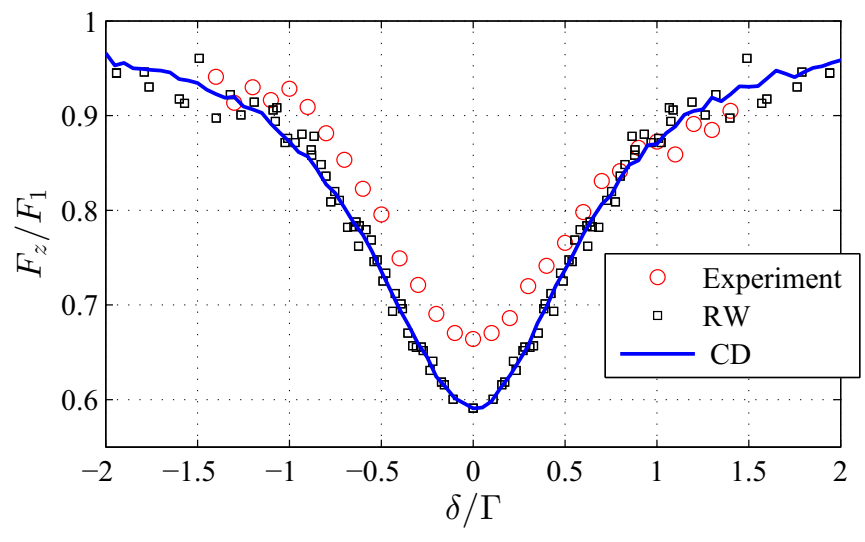

FIG. 2. (Color online) Normalized radiation pressure force acting on the center of mass of the atomic cloud as a function of the laser detuning $\delta$ (in unit of $\Gamma$ ), at constant saturation parameter $s(\delta)$. The experimental result for $b_{0}=2.19$ (red circles) are compared to the coherent CD model $\left(b_{0}=2.19\right.$, blue line $)$ and incoherent RW model (black squares) with corresponding optical thickness $b(\delta)$.

$5 \%$ accuracy and taking into account losses by the vacuum windows). The experimental value of the optical thickness $b_{0}$ is obtained by standard absorption imaging, using a linearly polarized laser beam. We note that such a transmission measurement is best described by atoms in a statistical mixture of the Zeeman sublevels in the ground state, with a corresponding average squared Clebsch-Gordon coefficient of $\left(2 F^{\prime}+1\right) / 3(2 F+1)=7 / 15$. The effective resonant optical thickness for a spherical Gaussian cloud of atoms distributed in a statistical mixture of the Zeeman sublevels is thus given by $b_{0}=\frac{2 F^{\prime}+1}{3(2 F+1)} \frac{3 N}{k \sigma_{R}^{2}}$, in contrast to a situation where all atoms would be pumped with a circularly polarized laser beam into the stretched state $\left|F=2, m_{F}=+2\right\rangle$.

In Fig. 2, we show the experimental result of the intrinsic radiation pressure force, proportional to the displacement of the center of mass of the atomic cloud (red spheres) as a function of the pushing beam detuning $\delta$, where we keep the saturation parameter $s=s_{0} /\left(1+4 \delta^{2} / \Gamma^{2}\right)$ constant. We clearly see an important reduction of the intrinsic radiation pressure force around the resonance. Note that with this experimental protocol, the mass of the atomic sample to be displaced as well as the single atom response are kept constant, allowing us to highlight the collective behavior.

We now turn to the comparison between the experimental data and our coherent and incoherent models. Momentum conservation arguments allow us to directly connect the farfield emission diagram of a sample to the momentum transfer to the center of mass of the sample. We stress that it is important not only to consider the shape of the emission diagram, but also the total scattered power, which can depend on the shape and opacity of the sample. Defining the intrinsic radiation pressure force along $\hat{e}_{z}$ by $F_{z}=(1 / N) \sum_{j} F_{j}$, where $F_{j}$ is the force acting on atom $j$ [16], we obtain the following relation:

$$
\frac{F_{z}}{F_{1}}=\frac{\sigma_{\text {tot }}}{N \sigma_{1}}\langle 1-\cos (\theta)\rangle,
$$

where $\cos \theta=\hat{\mathbf{k}} \cdot \hat{z}$ is the angle of the direction of emitted photon with the laser axis. We note that this relation holds 
both for the incoherent RW and coherent CD model. In (4), $F_{1}$ is the radiation pressure force for a single atom, and $\sigma_{\text {tot }}$ is the total scattering of the sample, which is different in the RW and the CD model. One can see from (4) that the emission diagram associated with the total power scattered from the incident beam allows us to predict the intrinsic force, proportional to the acceleration of the center of mass of the sample. The numerical results of our CD solution (blue line) also show a strong reduction of the force. To come forward with a simple interpretation of these results, one can associate the term corresponding to $\frac{\sigma_{\text {tot }}}{N \sigma_{1}}$ as a "shadow" effect, where that part of the force is obtained with only taking into account the attenuation of the incident laser beam. The recoil due to the rescattered photons is then properly taken into account by the last term of (4): $-\frac{\sigma_{\text {tot }}}{N \sigma_{1}}\langle\cos (\theta)\rangle$, related to the emission diagram of the photons. As shown in Fig. 2 the experimental result compares reasonably well both the $\mathrm{CD}$ and RW model when using the optical thickness evaluated for a statistical mixture of Zeeman sublevels, assumed to be a good approximation under multiple-scattering conditions, when the local polarization is well approximated by a field with random polarization.

\section{DISCUSSION}

In order to probe coherence effects under multiplescattering conditions, it is important to check to what extent an incoherent model also explains similar features. When looking at the prediction of the incoherent model, we find that there is no significant difference in the regime of parameters studied. This very close match between the incoherent and coherent models can be explained by the fact that the emission diagram only differs at two angles. In the backward direction, the coherent backscattering cone should result in a small increase of the intrinsic radiation pressure force in the coherent model compared to an incoherent model. However, the enhancement factor in the backward direction is less than 2 and the angular range of enhanced backscattering is very small for a dilute sample of atoms. On the other hand, the more important emission intensity in the forward lobe does not result in a significant change in the intrinsic radiation pressure force, as in this direction $(\theta \approx 0)$ the momentum transfer to the atoms is vanishing: $\langle 1-\cos (\theta)\rangle \approx 0$. It thus turns out that under these conditions, the intrinsic radiation pressure force is not a good measure to detect differences between coherent and incoherent scattering. We further investigate the range of validity of the RW model in Fig. 3, where we plot the intrinsic force extracted from the experimental data for various $b_{0}$, the RW simulation, and the CD simulation as a function of $b(\delta)$. The RW simulation is by definition a function of $b(\delta)$ only. A close inspection of the experimental and CD result shows small deviations from the RW model, including a red or blue asymmetry, visible at small $b(\delta)$. The origin of this asymmetry remains unknown. However, we observed it on $\mathrm{CD}$ data for much higher $b_{0}$ than experimentally measured. When looking for coherence effects, one does indeed expect to find cooperative signatures for large $b_{0}$ and large detunings, where a mean-field approach is assumed to be valid [26]. Another feature not included in the RW model are Mie resonances expected in the CD model for large $b_{0}$ [27]. We also note a small deviation of the experimental results compared to both the RW and CD model

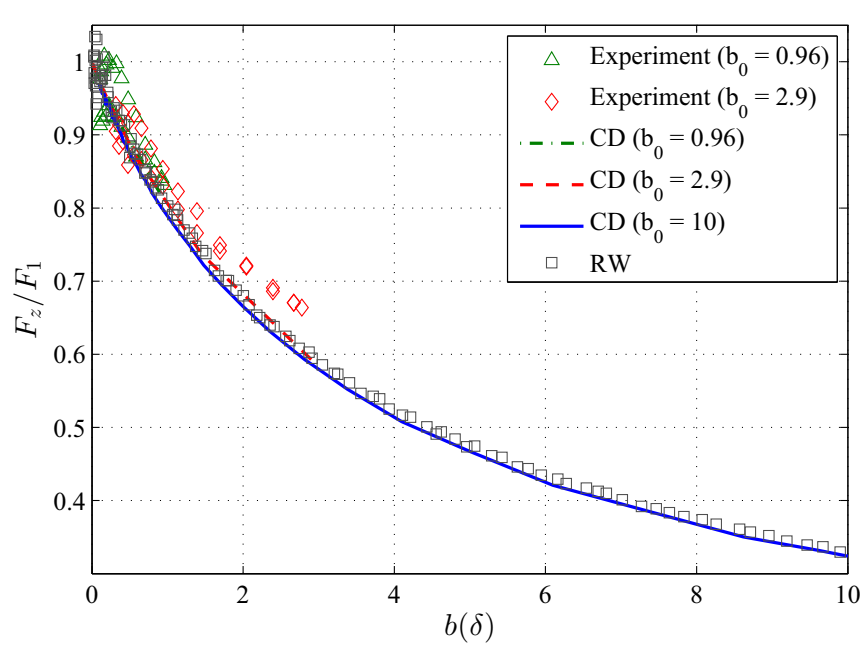

FIG. 3. (Color online) Normalized radiation pressure force as a function of the optical thickness. The experimental data for $b_{0}=0.96$ (green triangles) and $b_{0}=2.9$ (red diamonds) are compared to the results of the CD simulations, obtained for $b_{0}=0.96$ (green dashdotted line), $b_{0}=2.9$ (red dotted line), and $b_{0}=10$ (blue plain line), and of the RW model (gray squares). For the experimental data and the CD model, $b(\delta)$ is varied at fixed $b_{0}$ by changing the detuning. The $\mathrm{CD}$ data are superimposed as $b(\delta)$ appears as a universal parameter, and fluctuations are very small.

close to resonance ( $\delta=0)$ (see Figs. 2 and 3). This difference would correspond to a slightly smaller atom-light coupling than obtained from a statistical mixture of atoms in the Zeeman sublevels, which would however be rather surprising.

Finally, we plotted in Fig. 4 various data extracted from our CD simulation using the same Gaussian sphere that gives the emission diagram plotted in Fig. 1. Figure 4(a) represents the attenuation $e^{-b_{0}}$ of the incident laser field at the position $\hat{e}_{z}$ and along the transverse direction $\hat{e}_{r}$. Figure 4(b) represents the dipole excitation relative to the single atom limit $\left|\beta_{j}\right|^{2} /\left|\beta_{1}\right|^{2}$. For an integrated opacity $b_{0}>1$ (white line), one expects the atomic dipoles to be less excited by the incident laser field. Those atoms in the shadow are indeed significantly less excited. Figure 4(c) represents the phase of the atomic dipoles. Most of the atomic cloud has a phase $\phi=0$ corresponding to the laser field. In other words, almost the entire cloud is synchronized with the incident laser field even for $b_{0}>1$. This is somewhat in contradiction to what one would expect from a RW hypothesis, where in the multiple-scattering regime the phase is randomized after few scattering events and interference effects are smeared out by the disorder. This result is however in good agreement with the Ewald-Oseen extinction theorem [28] which specifies that the radiation from the atoms exactly cancels the electromagnetic field of the incident laser beam and replaces it by a field with a speed $c / n$ where $n$ is the index of refraction of the medium. As shown in [28], not only is the surface layer of the atomic cloud synchronized but the entire cloud participates to the cancellation of the incident field. This is in contrast with the common belief that optically thick samples are well described by the RW approach where the phase of a photon is randomized after few diffusion events, but is in agreement with related work [29,30] where a small persisting coherent component in the forward scattered 

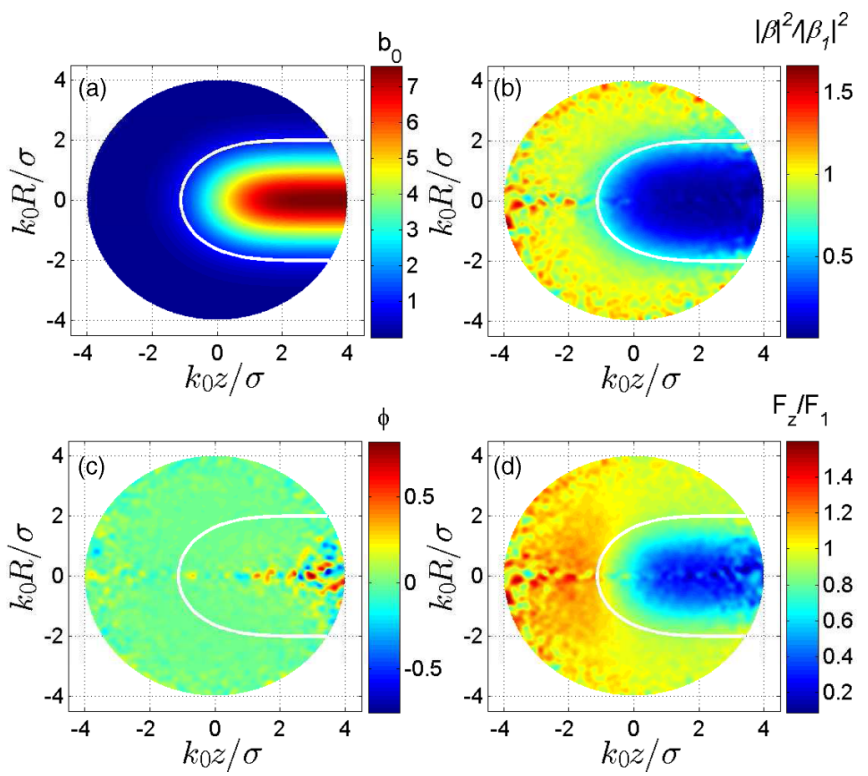

FIG. 4. (Color online) (a) Local optical thickness $b_{0}$ seen by the laser field propagating along the direction $\hat{e}_{z}$. The white line represents the multiple-scattering limit $b_{0}=1$. (b) Excitation amplitude of the atomic dipole compared to the single atom limit $\left|\beta_{j}\right|^{2} /\left|\beta_{1}\right|^{2}$. (c) Phase of the atomic dipole $\phi_{j}$. $\phi=0$ corresponds to the laser field phase corrected from its propagation along $\hat{e}_{z}$. (d) Local force compared to the single atom case $F_{z} / F_{1}$. All data are computed for a Gaussian sphere with $b_{0}=8$.

field has been reported. Figure 4(d) represents the local force. Although the atoms at the entrance of the cloud undergo a force larger than the single-atom force, those in the shadow are subjected to a lower force, resulting in a total force below the single-atom one, in agreement with the measurement reported in Fig. 3.

\section{CONCLUSION}

In summary, we have compared an incoherent and a coherent model for multiple scattering. The most prominent differences are the well-known coherent backscattering cone and an important forward lobe. We have compared the predictions of these two models to the experimental result of the intrinsic radiation pressure force acting on the center of mass of the atomic cloud and found that this force is not a good candidate to detect coherence effects in multiple scattering. Using an effective coupling strength for the atom light coupling in multiple scattering, we find a satisfactory quantitative agreement between the experiment and the numerical model. It would be interesting to study how these results compare to previous theoretical and experimental results, where a mean-field approach in terms of single-photon superradiance has been used. A precise experimental study of how multiple scattering sets in and to what extent a RW model can explain the whole range from large to small optical thickness is a complex task, as one needs to take into account the various Zeeman sublevels of the rubidium atoms. We expect that alternative observables beyond average values of the center-of-mass displacement of the cloud, such as correlations in the scattered light or heating of the cloud, might be good candidates to look for features of cooperativity in scattering of light by cold atoms.

\section{ACKNOWLEDGMENTS}

R.K. acknowledges valuable discussions with D. Delande. We acknowledge financial support from IRSES project COSCALI, from USP/COFECUB (Project No. Uc Ph 123/11), and from GDRI nanomagnetism, spin electroncics, quantum optics, and quantum technologies. M.T.R. was supported by an Averroès exchange program. R.B. acknowledges support from the Fundação de Amparo à Pesquisa do Estado de São Paulo (FAPESP).
[1] M. P. Van Albada and A. Lagendijk, Phys. Rev. Lett. 55, 2692 (1985).

[2] P.-E. Wolf and G. Maret, Phys. Rev. Lett. 55, 2696 (1985).

[3] D. S. Wiersma, P. Bartolini, A. Lagendijk, and R. Righini, Nature (London) 390, 671 (1997).

[4] R. T. F. Scheffold, R. Lenke, and G. Maret, Nature (London) 398, 206 (1999).

[5] D. S. Wiersma, P. Bartolini, A. Lagendijk, and R. Righini, Nature (London) 398, 207 (1999).

[6] A. Lagendijk, B. van Tiggelen, and D. S. Wiersma, Phys. Today 62(8), 24 (2009).

[7] T. Sperling, W. Buehrer, C. M. Aegerter, and G. Maret, Nat. Photon. 7, 48 (2012).

[8] F. Scheffold and D. Wiersma, Nat. Photon. 7, 934 (2013).

[9] G. Maret, T. Sperling, W. Bührer, A. Lubatsch, R. Frank, and C. M. Aegerter, Nat. Photon. 7, 934 (2013).

[10] B. A. van Tiggelen, A. Lagendijk, and A. Tip, J. Phys.: Condens. Matter 2, 7653 (1990).

[11] T. Karpiuk, N. Cherroret, K. L. Lee, B. Grémaud, C. A. Müller, and C. Miniatura, Phys. Rev. Lett. 109, 190601 (2012).

[12] F. Scheffold and G. Maret, Phys. Rev. Lett. 81, 5800 (1998).
[13] V. Krachmalnicoff, E. Castanié, Y. De Wilde, and R. Carminati, Phys. Rev. Lett. 105, 183901 (2010).

[14] H. Liu and P. Lalanne, Nature (London) 452, 728 (2008).

[15] R. H. Dicke, Phys. Rev. 93, 99 (1954).

[16] P. W. Courteille, S. Bux, E. Lucioni, K. Lauber, T. Bienaimé, R. Kaiser, and N. Piovella, Eur. Phys. J. D 58, 69 (2010).

[17] J. Hald, J. L. Sørensen, C. Schori, and E. S. Polzik, Phys. Rev. Lett. 83, 1319 (1999).

[18] B. J. Bloom, T. L. Nicholson, J. R. Williams, S. L. Campbell, M. Bishof, X. Zhang, W. Zhang, S. L. Bromley, and J. Ye, Nature (London) 506, 71 (2014).

[19] P. de Vries, D. V. van Coevorden, and A. Lagendijk, Rev. Mod. Phys. 70, 447 (1998).

[20] R. Pierrat, B. Grémaud, and D. Delande, Phys. Rev. A 80, 013831 (2009).

[21] S. Chandrasekhar, Radiative Transfer (Dover, New York, 1960).

[22] A. A. Svidzinsky, J. T. Chang, and M. O. Scully, Phys. Rev. A 81, 053821 (2010).

[23] T. Bienaimé, T. Bachelard, J. Chabé, M. T. Rouabah, L. Bellando, P. W. Courteille, N. Piovella, and R. Kaiser, J. Mod. Opt. 61, 18 (2014). 
[24] G. Labeyrie, F. de Tomasi, J.-C. Bernard, C. A. Müller, C. Miniatura, and R. Kaiser, Phys. Rev. Lett. 83, 5266 (1999).

[25] G. G. Padmabandu, C. Oh, and E. S. Fry, Opt. Lett. 17, 169 (1992).

[26] T. Bienaimé, S. Bux, E. Lucioni, P. W. Courteille, N. Piovella, and R. Kaiser, Phys. Rev. Lett. 104, 183602 (2010).
[27] R. Bachelard, P. W. Courteille, R. Kaiser, and N. Piovella, Europhys. Lett. 97, 14004 (2012).

[28] V. C. Ballenegger and T. A. Weber, Am. J. Phys. 67, 599 (1999).

[29] C. Yang, K. An, L. T. Perelman, R. R. Dasari, and M. S. Feld, J. Opt. Soc. Am. A 16, 866 (1999).

[30] D. Mackowski and M. Mishchenko, J. Quant. Spectrosc. Radiat. Transfer 123, 103 (2013). 